\title{
The discovery of novel CK1 delta inhibitors for bladder cancer therapy
}

\section{Chun-Han Chen}

\author{
Department of Pharmacology, Taipei Medical University, Taiwan
}

Bladder cancer is a common malignancy worldwide, but targeted therapy other than immune checkpoint inhibitors is currently unavailable. Hence, there is an unmet medical need for the development of new therapeutic approach to bladder cancer patients. Oncomine database revealed that gene expression of CK1 delta is higher in bladder cancer compared to normal tissue, and correlates with survival rate. The aims of this study is to investigate the role of CK1 delta in the progression of bladder cancer, and the feasibility of novel CK1 delta inhibitors as a therapeutic target. Lentiviral delivery of shRNA and CK1 delta inhibitors were used to modulate gene expression or kinase activity of CK1 delta in bladder cancer cell lines. Cell viability, cell cycle progression, apoptosis, migration and downstream signaling pathway were analyzed. The results showed that knocking down CK1 delta or treatment of CK1 delta inhibitors decreased cell viability and inhibited wnt/\&\#61538;-catenin pathway in bladder cancer cell lines. CK1 delta inhibitors increased the accumulation of sub-G1 phase by flow cytometry analysis, as well as the activation of caspase-3, 8, 9 and PARP cleavage by western blot, indicating the promotion of cell apoptosis. Knocking down of CK1 delta and the treatment of CK1 delta inhibitors impaired cell migration activity, and altered protein expression of EMT markers, such as E-cadherin, vimentin, snail and slug. In conclusion, this study demonstrates that CK1 delta is a potential target for bladder cancer therapy. 